\title{
Quality Assurance Improvements in Australian University Libraries
}

\section{Karen Tang,}

Associate Director Corporate Service,

\author{
Curtin University Library,
}

Western Australia

\section{Introduction}

In 2005 the Libraries of the Australian Technology Network (LATN) initiated a project to review current practice in quality assurance across its member libraries, draw out examples of best practice, and identify gaps and possible areas for improvement within their libraries. The project was intended as a collaborative, information sharing exercise that could facilitate continuous improvement in quality assurance in the libraries.

In 2009/2010 LATN members were re-surveyed to investigate whether changes had occurred in the intervening period, including what improvements had been made and where there were still gaps.

This paper examines the changes which had taken place in the libraries in the intervening period. Reports examining the full range of changes which had occurred are available elsewhere: Tang and Levinge (2006a), Tang and Levinge (2006b), Coen (2010) and Tang (2010). This paper focuses on developments in three aspects of the management of quality assurance within the libraries: responsibility for quality assurance; the use of quality, planning and/or performance frameworks within the libraries; and individual work planning and training for quality. These aspects have been selected as they form part of the criteria used by Wilson and Town (2006) to assign libraries to a quality maturity level. The paper is therefore a consideration of the extent to which there has been growth in the quality maturity of the participating libraries over the past six years.

While the paper is based on the experience of Australian and New Zealand libraries, it addresses concerns and provides solutions which are appropriate internationally. It provides a range of options which an individual library could adopt depending on its own context.

\section{The "Quality Maturity Model"}

When embarking on the $2005 / 6$ benchmarking review of LATN members' quality assurance practices, the reviewers found that an accepted scheme against which members' performance on quality assurance could be evaluated was lacking. In the United Kingdom, Wilson and Town (2006, p. 75) similarly found that an appropriate framework for assessing the quality level of libraries was not present in the literature. They noted that "there is much in the literature ... about frameworks for measuring the quality of a product, process or service, but no framework for measuring the quality of the quality-process" (Wilson and Town (2006, p. 76). In the absence of an agreed framework, Wilson and Town developed 
their own Library Quality Maturity Model which measured a library's quality maturity on a five-step scale, from Level 1 - Initial, to Level 5 - Optimising :

Specific attributes were associated with each level. For example, at Level 1:

- quality is achieved in an ad hoc way;

- customer satisfaction is reactive and unpredictable;

- quality depends on the capabilities of individuals, and varies with their innate skills, knowledge and motivations; and

- training for quality is $a d$ hoc and reactive to an ability to undertake a specific task adequately.

By contrast, at Level 5:

- the entire organisation is focussed on continuous improvement in every service, product and process;

- all staff are encouraged to continuously improve themselves and their work;

- the organisation is able to identify weaknesses, and the means to strengthen the process, proactively with the goal of preventing problems;

- innovations that exploit the best practices are identified and transferred throughout the organisation; and

- training for quality is focussed on preparing staff for future organisational requirements.

Wilson and Town (2006, pp. 77-78)

\section{Methodology for the LATN reviews}

The author led the conduct and analysis of two surveys of the members of the LATN group.

- A 2005/6 study reviewed quality assurance practices at the member libraries, to draw out examples of best practice and identify gaps and possible areas for improvement within the libraries. It was based on a review of member libraries' websites, a questionnaire completed by a nominee from each member library, and follow-up inperson interviews with each nominee and the University Librarian of each institution.

- In 2009/2010 the same questionnaire was re-administered to investigate whether changes had occurred in the intervening period, including what improvements had been made and where there were still gaps. Had members made improvements to their quality assurance processes based on the findings of the first study or for other reasons? Due to restructuring and staffing changes at most libraries, with one exception the questionnaire was completed by a different nominee in $2009 / 10$ than 2005. No interviews or site visits were conducted in 2009/10.

(Hereafter, for simplicity the studies will be referred to as the 2006 and 2010 reviews, although in both cases they commenced a year earlier.)

To elicit additional information, follow-up email exchanges were carried out in 2011.

A broad view of "quality assurance" was taken for the reviews including planning and performance measurement; use of frameworks, charters and service level agreements; benchmarking and evaluation. Responsibilities, training and communication in relation to 
quality were considered, together with the extent to which it could be said that quality was embedded in the culture of the library.

Participating libraries comprised:

- Auckland University of Technology in Auckland, New Zealand

- Curtin University of Technology in Perth, Australia

- Queensland University of Technology in Brisbane, Australia

- Royal Melbourne Institute of Technology University in Melbourne, Australia

- University of South Australia in Adelaide, Australia

- University of Technology Sydney in Sydney, Australia.

\section{Approach of the current paper}

The 2006 and 2010 LATN reviews did not attempt to assign the participating libraries to a level of Wilson and Town's (2006) Quality Maturity Model. However, reflecting on the changes observed since 2006, and using the attributes associated with the various levels of Wilson and Town's model, it could be argued that a maturing of quality assurance amongst some of the libraries has occurred.

The present paper examines this maturation by examining three of the criteria used by Wilson and Town (2006) to assign libraries to a quality maturity level:

- responsibility for quality assurance

- the use of quality, planning and/or performance frameworks within the libraries

- individual work planning and training for quality

\section{Australian university environment $2005-2011$}

Before considering the three aspects noted above in detail, it is necessary to note some of the changes which occurred in the Australian university environment between the LATN reviews.

This period saw a continuation in the growing competition between Australian universities and other higher education providers (national and international), and between Australian universities themselves (Yerbery in Williamson 2006, p. 549). This competition was coupled with a desire among many universities, including members of the Australian Technology Network, to be recognised not only or primarily for their teaching but for their research. In such an environment comparisons, benchmarking and rankings are of vital importance, to identify and/or publicise how the university is performing vis-a-vis competitors.

The government "reviewathon" of 1995 - 2005 noted by Yerbery (including a predominance of reviews, regulation and reporting) has also continued, despite a change of government in 2007. Under the current Australian government, there has been a particular emphasis on making comparative quantitative information on education publicly available. A somewhat controversial "My School" website has been launched and a "My University" site is planned for 2012. 
During the period all but one of the parent universities of the members of LATN underwent at least one quality assurance audit by the Australian Universities Quality Agency, established in 2002 by the Australian Government as the national quality assurance agency in higher education. For their audits, universities were required to submit portfolios documenting their approach to quality assurance. During their visits, the AUQA auditors interviewed large numbers of staff and students. Preparations for such audits have focussed universities on their quality assurance documentation, processes and training.

In 2007 the previous government introduced Higher Education Workplace Relations Requirements compliance as condition of funding increases. One of the HEWR requirements was the existence of performance management schemes for university staff.

In such an environment, quality maturation could be expected.

\section{Findings}

In 2005/6:

- only one library had a specific person responsible for quality,

- only two had a documented quality framework and

- there was a gap across the whole group in individual planning and training for quality.

By 2011:

- three or four have a specific person responsible for quality,

- all six have a documented quality framework and

- all except one do individual work planning and training for quality.

\section{$\underline{\text { Responsibility for quality assurance }}$}

In 2006 the reviewers found three models for responsibility for quality in libraries; centralised (with a designated quality officer), within a manager's portfolio, or devolved where all managers are responsible for implementing quality within the library. While no model was considered to be perfect, the reviewers argued that a centralised model at the early stages of development of a quality assurance program gave a dedicated driver to the development and implementation of the program. Once a quality program was in place more devolution could take place.

In 2006 one library had adopted a centralised model by appointing a designated Quality Librarian and one library was in the process of appointing a Quality Co-ordinator. By 2010 three libraries had appointed or designated a specific quality officer/librarian, while the library which had previously had a Quality Librarian in the initial stages of its quality development now believed its program was sufficiently mature that it could be allocated, together with other corporate functions to one of the library's operational units. This library feels the quality program initially established by its quality officer has now been in operation sufficiently long that the key policies, procedures and documents are in place. The documents require only maintenance and updating and periodic review. The quality cycle is established as part of the library's annual timetable so that responsibility for various activities can be shared without the danger that some activities will be overlooked. The culture of the management team has been changed so that, without prompting, managers adopt quality approaches in 
what they do and expect the same of their colleagues, so that quality no longer requires a specific catalyst or champion.

Titles and responsibilities of the central quality officer varied, for example: Quality, Planning \& Research Librarian; Co-ordinator Project Management and Quality Assurance; Planning and Quality Co-ordinator; Quality and Planning Manager. Some were full-time, others part time. Some required qualifications in librarianship, others qualification in information management, business or management.

All job descriptions for the central quality officer placed an emphasis on co-ordination of the library's quality activities. All included planning, collation and analysis of statistics and performance measures, conduct of client evaluations, encouragement of best practice or continuous improvement, internal and external reporting and development of documentation.

Those libraries which had specific quality officers valued them. One library particularly appreciated having some designated time for quality assurance by a specifically designated person.

\section{Use of quality, planning or performance frameworks}

In 2006 one library had a functioning overall quality framework and one library had separate planning and performance frameworks. Other libraries reported use of the PDRI (Plan-DoReview-Improve) cycle or used "elements of a quality framework" without an integrating document. Most had library plans but the outlook for some of these was only one year, and the plans did not always include quantitative targets or indicators of success.

Today this landscape had changed significantly. All libraries have a framework or have adopted the approach to quality required by their parent institutions.

The libraries which had adopted a quality framework and planning/performance frameworks in 2006 were ahead of their universities in these developments: when their universities subsequently developed frameworks, the libraries had had to adapt or overhaul theirs to ensure compliance with university requirements or integration with higher level approaches.

Two libraries have developed text documents outlining and linking the library's planning, performance and benchmarking frameworks or strategies. At one library, this document also explains the relationship between the library's approach to quality and that of the parent university. This university uses the Australian Business Excellence Framework and the Balanced Scorecard.

Other libraries have simply adopted the framework documents of their parent university, or a part of them. One for example uses a planning framework and an indicator framework, and a quality management system to continuously improve the plans and indicators. Another uses a balanced scorecard approach to planning and performance, "harmonising" its own scorecard with those of other units in the university's academic portfolio (including student services and learning and teaching units). A third library uses its university's "action planning template" which emphasises the four-step Plan-Do-Review-Improve (PDRI) cycle adapted from the Approach-Deployment-Review- Improvement (ADRI) approach. At this library, staff 
from the university's planning and quality unit conducted a workshop to ensure that area plans were explicitly linked to the university plan.

The library which in 2006 had a quality framework has now discontinued it. This library now refers to the quality assurance cycle of its parent university and the service management framework of the division (faculty) of which it forms a part. The library's strategic plan is now no longer based on the balanced scorecard but on its university's "blueprint" strategic planning document.

All libraries expressed the view that having a documented quality framework was useful in assuring quality in their library. The quantitative and cyclical nature of quality frameworks are key here, with libraries now being committed to developing plans with associated quantitative measures of success, to revisiting their plans and performance indicators regularly, and to reporting internally or externally on the extent to which targets are being met, and taking corrective action where necessary. Many of the quantitative measures incorporated in the frameworks are client satisfaction measures or measures which the library believes will result in client satisfaction.

\section{Quality responsibility and frameworks in Quality Maturity Model}

The appointment of quality officers and development of documented quality processes has clearly moved the LATN libraries beyond Wilson and Town's (2006) Level 1 of Quality Maturity characterised by individualism, ad hoc-ery and undefined processes:

Level 1 - initial. The quality management process is ad hoc, and occasionally even chaotic. Few processes are defined, and success depends on individual effort and heroics .... [Q]uality depends on the capabilities of individuals, and varies with their innate skills, knowledge and motivations Wilson and Town (2006, p. 77)

Through the work of the quality officers with their libraries' management teams and the adoption of quality frameworks, the LATN libraries are all at least at Level 2 of Quality Maturity:

Level 2 - repeatable. Basic quality management processes are established. The necessary management processes are in place to repeat earlier quality levels... [Q]uality policies and procedures to implement these policies are established; ... such management processes are practised, documented, enforced... Wilson and Town (2006, p. 77)

Most importantly processes are repeated, so that the libraries can build on earlier success or identify and make improvements as required.

It could be argued that one or two of the libraries are moving towards Level 3 quality maturity:

Level 3 - defined. The quality processes are documented and standardised. All work derives from the organisational strategy. Wilson and Town (2006, p. 77)

The existence of a "defined, documented, organisational strategy, from which all work processes are derived" is however but one of the characteristics of Level 3 quality maturity. Other requirements are "an organisation-wide understanding of the activities, roles and 
responsibilities of each member of the library staff and how they fit into the organisational strategy" and "training for quality is a cycle of training needs assessment and programme provision" (Wilson and Town 2006, pp. 77-78). To what extent are the LATN libraries exhibiting these characteristics?

\section{Individual work planning and training for quality}

One of the most marked changes between 2006 and 2010 was the growth in individual work planning and performance review. In 2006 this was identified by the LATN reviewers as a gap across the network; by 2010 all but one library had an individual work planning and performance review process in place. Ideally use of such plans and assessments should assist in taking quality beyond library management, to develop amongst all staff an awareness and culture of continuous improvement, and an alignment between the staff member's efforts and the strategic direction of their team, library and/or university.

The schemes in place share much in common:

- a template based on a university-wide version

- an annual timeframe

- space for the staff member to list a number of objectives, goals or focus areas

- some indicators by which completion of the objectives will be determined or measured

- space for the staff member and their manager to record progress on/completion of the objective

- identification of the staff member's development needs, to facilitate the completion of the objectives or other career/work goals as set out in the document

Variations are also evident. Only on one library's form is the individual staff member specifically required to indicate a unit/library/university initiative or objective to which their proposed objective is related. However the guidelines to completing the forms of other libraries make it clear that alignment with university/library goals and directions is expected.

The connection of the individual work plan and performance review to the quality cycle and continuous improvement is implied in all but one of the approaches but made explicit in only a few. The guidelines for completing the template at one institution use a quality cycle with four phases (Prepare, Meet, Do, and Review, feedback \& support) to explain what is intended at each stage of the process.

It could be argued that use of individual work planning and performance measurement provides training for staff in quality, through familiarising staff with approaches such as PDRI/ADRI and continuous improvement and encouraging the use of targets and specific indicators of success and alignment of their actions with unit/library/university goals and strategies. If so, then the increased use of individual work planning and performance measurement can also be seen as sign of maturation of quality under Wilson and Town's (2006) model. Training for quality has moved from "ad hoc and reactive to an ability to undertake a specific task adequately" (Level 1), through "training for quality is provided as a programme of training for specific work tasks and/or is reactive to events" (Level 2) to 
"training for quality is a cycle of training needs assessment and programme provision" (Level 3). (Wilson and Town 2006, pp. 77-78)

Through their individual work planning schemes, the libraries are also moving to achievement of another characteristic of Level 3: "there is organisation-wide understanding of the activities, roles and responsibilities of each member of the organisation, and how they fit into the organisational strategy. (Wilson and Town 2006, p. 78)

\section{Conclusion}

Clearly then there have been improvements in quality assurance among LATN libraries since 2005. These are probably largely a consequence of the increased focus on quality in higher education in Australia during the corresponding period. However there has also been a contribution to the improvements through the libraries learning from each other through the LATN quality assurance benchmarking studies.

Wilson and Town's (2006) Quality Maturity Model has provided a framework within which to measure improvement to date. Clearly all the LATN libraries have some way to go before it could be argued that they have reached Level 5 quality maturity. However the beauty of Wilson and Town's model is that it gives libraries a roadmap for where they might go and what they need to aim for.

\section{References}

Coen, M 2010, Quality Assurance within ATN Libraries: a LATN Benchmarking Project. Descriptive Report. Accessed January 17, 2011. http://www.latn.edu.au/

Tang, K 2010, Quality Assurance within ATN Libraries: a LATN Benchmarking Project. Analysis of the Results of the 2006 and 2010 LATN Reviews. Accessed January 17, 2011. http://www.latn.edu.au/

Tang, K and Levinge, L 2006a, Quality Assurance within ATN libraries: a LATN Benchmarking Project. Interim Report. Accessed January 17, 2011. http://www.latn.edu.au/

Tang, K and Levinge, L 2006b, Quality assurance within ATN libraries: a LATN Benchmarking Project. Evaluative Report. Accessed January 17, 2011. http://www.latn.edu.au/

Williamson, V 2006, 'Surviving Change and Growing the Profession Together', Library Management, vol. 27, no. 8, pp. 548-561.

Wilson, F and JS Town 2006, 'Benchmarking and Library Quality Maturity', Performance measurement and metrics, vol. 7, no. 2, pp. 75-82. 